Pathologe 2014 · 35:191-195

DOI 10.1007/s00292-013-1790-1

Online publiziert: 22. Juni 2013

(c) Springer-Verlag Berlin Heidelberg 2013

Redaktion

K.W. Schmid, Essen

\author{
T. Hansen ${ }^{1} \cdot$ F. Hartmann ${ }^{2} \cdot$ W. Hiller ${ }^{3} \cdot$ M. Schütz ${ }^{4} \cdot$ I. Oschlies $^{5}$ \\ ${ }^{1}$ Institut für Pathologie, Klinikum Lippe GmbH, Detmold \\ ${ }^{2}$ Klinik für Onkologie und Hämatologie, Klinikum Lippe GmbH, Lemgo, Detmold/Lemgo \\ ${ }^{3}$ Klinik für Allgemein-, Viszeral- und Thoraxchirurgie, Klinikum Lippe GmbH, Detmold \\ ${ }^{4}$ Institut für Diagnostische und Interventionelle Radiologie, Klinikum Lippe GmbH, Detmold \\ ${ }^{5}$ Institut für Pathologie, Sektion Hämatopathologie und Lymphknotenregister, \\ Klinikum Schleswig-Holstein, Christian-Albrechts-Universität Kiel
}

\title{
Primäres Hodgkin-Lymphom des Kolons
}

\section{Kasuistik}

\section{Anamnese und klinischer Befund}

Eine 74-jährige Patientin stellte sich wegen unklarer Gewichtsabnahme (ca. $11 \mathrm{~kg}$ ) vor. Die Patientin berichtete ferner über zwischenzeitliche Blutabgänge mit dem Stuhl. Nachtschweiß oder Fieber wurden verneint. In der Vorgeschichte waren ein metabolisches Syndrom mit Diabetes mellitus II, Hyperlipidämie, Hyperurikämie sowie eine arterielle Hypertonie bekannt, ferner eine chronisch-obstruktive Lungenerkrankung bei Nikotinabsus (27 „packyears"), die zuletzt 2 Monate vor der aktuellen Aufnahme akut exazerbiert und therapiebedürftig war. Aus der Anamnese ergab sich zudem kein Hinweis auf eine maligne Vorerkrankung. Eine Immundefizienz oder die Einnahme immunsuppressiver Medikamente waren nicht bekannt.

Bei der körperlichen Untersuchung ergab sich kein richtungsweisender Befund. Laborchemisch fand sich der Hinweis auf eine mikrozytäreAnämie ( $\mathrm{Hb} 12,3 \mathrm{~g} / \mathrm{dl}$, Erythrozytenzahl 5,2/pl, Hkt 38\%, MCV $73,1 \mathrm{fl}$ ), ferner war die Leukozytenzahl im Normbereich (5,3/nl). Der Wert für die Laktatdehydrogenase war mit $284 \mathrm{U} / \mathrm{l}$ grenzwertig erhöht. Die übrigen Parameter waren bis auf eine Erhöhung der Harnsäure $(7,4 \mathrm{mg} / \mathrm{dl})$ ebenfalls unauffällig.

\section{Endoskopische und bildgebende Untersuchung}

Die Ösophagogastroduodenoskopie wies keine Blutungsquelle auf. Hingegen zeigte sich bei der koloskopischen Untersuchung in der rechten Flexur ein exulzerierter Tumor, der etwa die halbe Zirkumferenz einnahm und nicht stenosierend war (- Abb. 1). Aus diesem Areal wurden Biopsate für die histologische Untersuchung gewonnen. Hier fand sich lediglich hyperplastische Kolonmukosa mit Anteilen eines frischen Ulkus.

In einer zusätzlich durchgeführten Computertomographie des Abdomens mit Kontrastmittel zeigte sich im Colon ascendens eine langstreckige Kolonwandverdickung. Ferner fanden sich bis $9 \mathrm{~mm}$ große lokoregionäre Lymphknoten (- Abb. 2). In weiteren bildgebenden Verfahren (Sonographie Abdomen, Röntgen Thorax, CT Thorax) ergab sich kein Hinweis auf eine Tumormetastasierung. Der Fall wurde auf der interdisziplinären Tumorkonferenz besprochen, wobei eine operative Exploration festgelegt wurde. Daraufhin wurde eine Hemikolektomie rechts durchgeführt.

\section{Pathologischer Befund}

Das Operationspräparat wurde in 4\%igem Formalin fixiert. Makroskopisch fand sich im Colon ascendens ein $5,5 \times 3 \times 2 \mathrm{~cm}$ messender schüsselförmig gestalteter und zentral ulzerierter Tumor. Dieser zeigte eine weißliche Schnittfläche, das Tumorgewebe reichte bis in die Subserosa hin- ein (- Abb.3). Die histologische Aufarbeitung ergab einen diffus wachsenden Tumor mit einer gemischten lymphoiden Population, wobei sich neben Lymphozyten und Plasmazellen auch vermehrt und teilweise gruppiert angeordnete eosinophile Granulozyten nachweisen ließen. In Abschnitten war das Stroma kollagenfaserreich (• Abb. 4). Dazwischen fanden sich einzeln gelegene prominente mononukleäre Zellen mit vergrößerten Kernen und prominenten eosinroten bzw. metachromatischen Nukleolen, ferner auch immer wieder mehrkernige Riesenzellen, mit teilweise spiegelbildlich angeordneten Zellkernen und wiederum prominenten Nukleoli (• Abb.5).

Aufgrund des konventionell-histologischen Befundes ergab sich somit der Hinweis auf klassische Hodgkin- und Reed-Sternberg-Zellen. Immunhistochemisch zeigten diese Zellen eine Positivität für CD30 (• Abb. 6) und Negativität für CD45. Die Reaktion für CD15 fiel ebenfalls negativ aus. CD20 wurde arealweise schwach von den Hodgkin-Blasten exprimiert, wobei das Begleitinfiltrat sowohl B-Zell-reiche Abschnitte (CD20+) wie auch T-Zell-reiche Areale (CD3+) aufwies (letztere in unmittelbarer Nachbarschaft der Hodgkin- und Reed-Sternberg Zellen). Die weiterführende immunhistochemische Analyse im Rahmen der konsiliarischen Zweitbegutachtung in Kiel ergab die für Hodgkin-Lymphome typische schwache nukleäre Positivität für PAX-5 (- Abb.7) bei ansonsten stark herabreguliertem B-Zell-Immunphänotyp mit nur partieller schwacher Expres- 

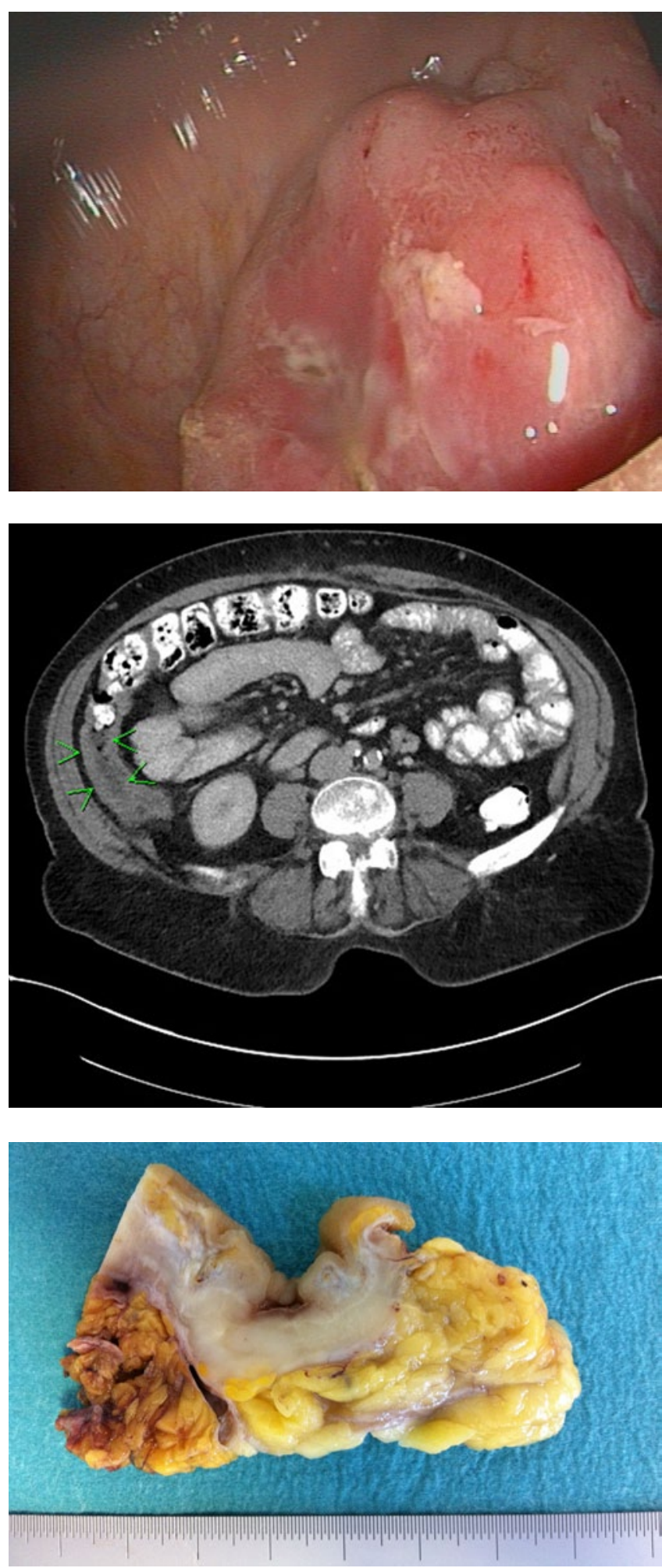

sion von CD20 sowie negativer Reaktion für CD19 und CD79a. Auch in der Untersuchung des Lymphomregisters konnte eine CD15-Expression nicht nachgewiesen werden. Darüber hinaus wurde in den Hodgkin-Blasten das Epstein-Barr-Virus (EBV) detektiert, sowohl mittels In-situ-
Abb. $1<$ Endoskopische Aufnahme des Tumors im Kolon

Abb. $2<$ Computertomographischer Befund der Wandverdickung im Kolon

Abb. $3<$ Makroskopischer Befund mit der grauweißen homogenen Schnittfläche des Tumors

\section{Diagnose und Verlauf}

Die weitere Untersuchung von 22 regionären Lymphknoten ergab keinen Lymphombefall. Auch in anschließenden Staginguntersuchungen konnte keine weitere Lymphominfiltration nachgewiesen werden. Somit ist der Befund abschließend als primäres Hodgkin-Lymphom des Kolons einzustufen. Postoperativ konnte die Patientin beschwerdefrei entlassen werden. Auf Empfehlung der GHSG-Studienzentrale Köln ist eine zusätzliche Gabe von 2 Zyklen ABVD-Chemotherapie (Adriamycin, Bleomycin, Vinblastin, Dacarbacin) ohne Bestrahlung aufgrund der vollständigen Tumorentfernung analog zur Niedrigrisikostratifizierung vorgesehen.

\section{Diskussion}

Der Anteil primärer Lymphome des Gastrointestinaltrakts an allen extranodalen Lymphomen beträgt 30-40\%. In der überwiegenden Mehrheit (bis zu 75\%) betreffen diese den Magen [2]. Primäre Lymphome des Kolons sind hingegen eine Seltenheit. In 2 nahezu zeitgleich erfolgten Analysen größerer Kohorten ergab sich ein Anteil der malignen Lymphome unter allen untersuchten Kolonneoplasien von lediglich 0,9 [4] bzw. 0,48\% [5]. Am häufigsten konnte ein Lymphombefall im Zökum dokumentiert werden (60\%), gefolgt von Colon ascendens mit rechter Flexur (27\%) und Sigma (13\%) [6]. Die weitaus überwiegende Mehrheit gehört zur Gruppe der Non-Hodgkin-Lymphome (NHL), während Hodgkin-Lymphome im Gastrointestinaltrakt viel seltener auftreten und speziell im Kolon bzw. Rektum nahezu ausschließlich in Form von Kasuistiken beschrieben wurden. Für die Diagnose eines primären kolorektalen Lymphoms gelten folgende Kriterien [10]:

- keine vergrößerten superfiziellen

Lymphknoten bei primärer

Präsentation,

- negativer Röntgen-Thorax-Befund insbesondere hinsichtlich mediastinaler Lymphknoten,

(EBER, - Abb. 8) als auch über Nachweis einer Expression des LMP-Proteins. Abschließend wurde eine transmurale Infiltration der Kolonwand durch ein klassisches Hodgkin-Lymphom diagnostiziert.
- normaler Knochenmarkbefund, normale Leukozytenzahl,

- bei der Laparotomie sind lediglich die regionären Lymphknoten auffällig, 
- Leber und Milz sind makroskopisch tumorfrei.

Neuerdings werden diese eher strikten Kriterien hinterfragt [10]. Im hier präsentierten Fall konnten jedoch alle Punkte bestätigt werden, was zur abschließenden Diagnose des Tumors als primäres Hodgkin-Lymphom des Kolons führte.

Bezüglich der klinischen Symptomatik ist auffallend, dass die Beschwerden unspezifisch sind, was mit einem prolongierten Krankheitsverlauf einhergehen kann [11]. Serologische Befunde, welche auf ein Lymphom hinweisen können, fehlen beim primären kolorektalen Lymphom häufig, sodass die klinische Diagnostik zusätzlich erschwert ist. Wie der vorliegende Fall zudem zeigt, können Biopsate auch, speziell wenn es sich um oberflächliche Proben aus exulzerierten Lymphomen handelt, falsch-negativ sein.

Wichtig für die Diagnosestellung des klassischen Hodgkin-Lymphoms sind die typische Histomorphologie mit Nachweis klassischer Hodgkin-Blasten, das typische gemischtzellige Begleitinfiltrat und der Nachweis des charakteristischen Immunphänotyps der Hodgkin-Zellen mit CD30-Expression, variabler Expression von CD15, variabler EBV-Assoziation sowie dem Nachweis eines „herabreguliertem“ B-Zell-Profils. Der B-Zell-Transkriptionsfaktor PAX-5 ist mit einer meist charakteristisch nukleären schwachen Anfärbung in fast allen Hodgkin-Lymphomen als Hinweis auf eine B-Zell-Differenzierung nachweisbar und dient auch der differenzialdiagnostischen Abgrenzung gegenüber anderen CD30-exprimierenden Neoplasien. Klassische B-ZellMarker wie CD20 sind hingegen meist negativ oder nur schwach exprimiert. In den seltenen Fällen mit starker CD20-Expression müssen differenzialdiagnostisch das lymphozytenprädominante HodgkinLymphom sowie andere B-NHLs ausgeschlossen werden [9]. Der hier publizierte Fall (CD30+, CD20-/+, EBV+, PAX-5 $-/+$, CD15-) erfüllte somit alle typischen Kriterien des klassischen Hodgkin-Lymphoms, hier EBV-assoziiert. Die fehlende Expression von CD15 ist kein Gegenargument und wird bei ca. $40 \%$ sonst klassischer Hodgkin-Lymphome beobachtet [9].

Pathologe 2014 · 35:191-195 DOI 10.1007/s00292-013-1790-1

(c) Springer-Verlag Berlin Heidelberg 2013

\section{T. Hansen · F. Hartmann · W. Hiller · M. Schütz $\cdot$ I. Oschlies Primäres Hodgkin-Lymphom des Kolons}

\section{Zusammenfassung}

Primäre Hodgkin-Lymphome stellen im Kolon eine Rarität dar. Wir berichten über den Fall einer 74-jährigen Patientin, die sich wegen Gewichtsverlust und Blut im Stuhl vorgestellt hatte. Bei der endoskopischen Untersuchung zeigte sich ein blutender Tumor in der rechten Kolonflexur, welcher aber bioptisch nicht als maligne verifiziert werden konnte. Interdisziplinär fiel dennoch die Entscheidung für ein exploratives operatives Vorgehen. Im Hemikolektomiepräparat rechts fand sich ein 5,5 cm großer zentral ulzerierter Tumor, welcher histologisch als klassisches Hodgkin-Lymphom diagnostiziert wurde. Ein Lymphknoten- oder ein anderer Organbefall ließen sich in weiteren Staginguntersuchungen nicht belegen, sodass die Kriterien für ein primäres Hodgkin-Lymphom des Kolons erfüllt wurden. Die weiterführende Diagnostik eines primären Kolonlymphoms kann erschwert sein, da die Klinik typischerweise uncharakteristisch ist und speziell bei ulzerierten Tumoren auch der bioptische Nachweis negativ ausfallen kann.

\section{Schlüsselwörter}

Ulzerierter Tumor - Epstein-Barr-Virus (EBV) . Immunhistochemie - Lymphomregister · Chemotherapie

\section{Primary Hodgkin's lymphoma of the colon}

\section{Abstract}

Primary Hodgkin's lymphoma of the colon is exceedingly rare. We report on the case of a 74-year-old female patient presenting with weight loss and hematochezia. Proctocolonoscopy revealed a bleeding tumor localized in the right colonic flexure. Histological examination of initial mucosal biopsies could not verify malignancy; however, explorative surgery was decided in an interdisciplinary conference setting and right-sided hemicolectomy was performed. Macroscopically, a tumor measuring $5.5 \mathrm{~cm}$ in maximum diameter was found. By means of histology and immunohistochemistry the diagnosis of classic Hodgkin's lymphoma was made. Mesenteric lymph nodes were not affected and postoperative staging revealed no systemic spread. Therefore, the tumor fulfilled the criteria of a primary colonic Hodgkin's lymphoma. Diagnosis of primary colonic lymphoma can be difficult as clinical symptoms are typically unspecific and, as shown in this case, even primary biopsy histology can be falsely negative.

\section{Keywords}

Ulcerated tumor - Epstein-Barr virus (EBV) . Immunohistochemistry - Lymphoma register . Chemotherapy

\section{Differenzialdiagnosen}

Im hier vorliegenden sehr seltenen Fall eines primär intestinalen EBV-assoziierten Hodgkin-Lymphoms bei einer älteren Patientin sind 2 Differenzialdiagnosen näher zu diskutieren:

$$
\begin{aligned}
& \text { Abgrenzung gegenüber einer so } \\
& \text { genannten „EBV-assoziierten } \\
& \text { mukosalen Ulzeration“ [3], } \\
& \text { - Abgrenzung gegenüber einem } \\
& \text { „EBV-positiven diffus großzelligen } \\
& \text { Lymphoms des älteren Patienten“ [8]. }
\end{aligned}
$$

Die „EBV-positive mukokutane Ulzeration" ist eine relativ neu beschriebene Entität und entspricht einer EBV-assoziierten mukosalen Lymphoproliferation, welche histomorphologisch und auch immunhistochemisch Infiltraten eines klassischen Hodgkin-Lymphoms extrem ähnlich sein kann, häufig im Rahmen einer Immunsuppression auftritt und einen klinisch blanden, selbstlimitierenden Verlauf nimmt [3]. Allerdings ist diese Erkrankung definitionsgemäß als oberflächliche Schleimhautulzeration beschrieben, eine transmurale tumorbildende Infiltration wie im hier publizierten Fall ist mit dieser Diagnose nicht vereinbar. Da „EBV-positive mukokutane Ulzerationen" häufiger im Rahmen einer Immunsuppression auftreten, ist es möglich, dass ein Teil der publizierten Fälle zu intestinalen Hodgkin-Lymphomen bei Patienten mit chronisch entzündlichen 


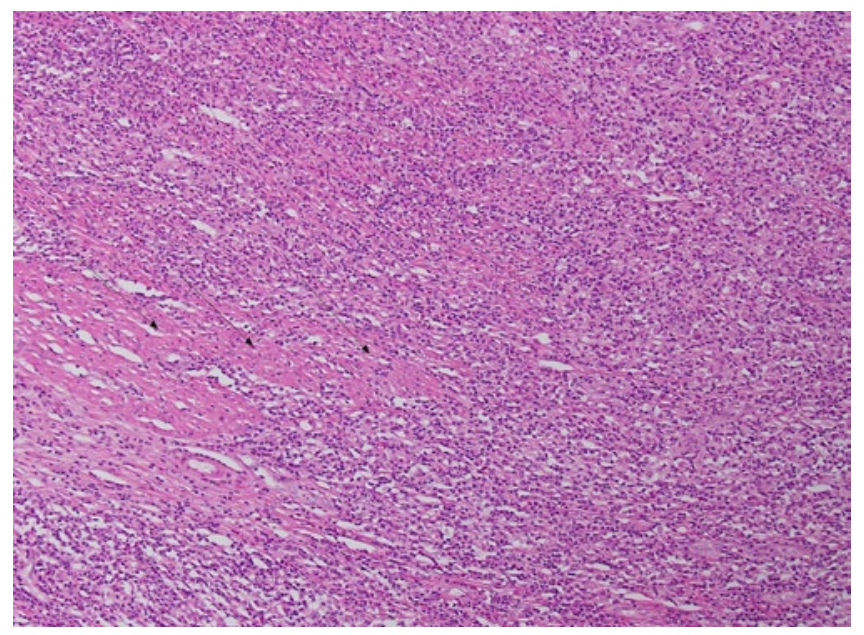

Abb. $4<$ Histologische Übersicht mit Nachweis der Infiltration zwischen bestehenden glattmuskulären Strukturen (Pfeile, HE-Färbung, Vergrößerung 100-fach)

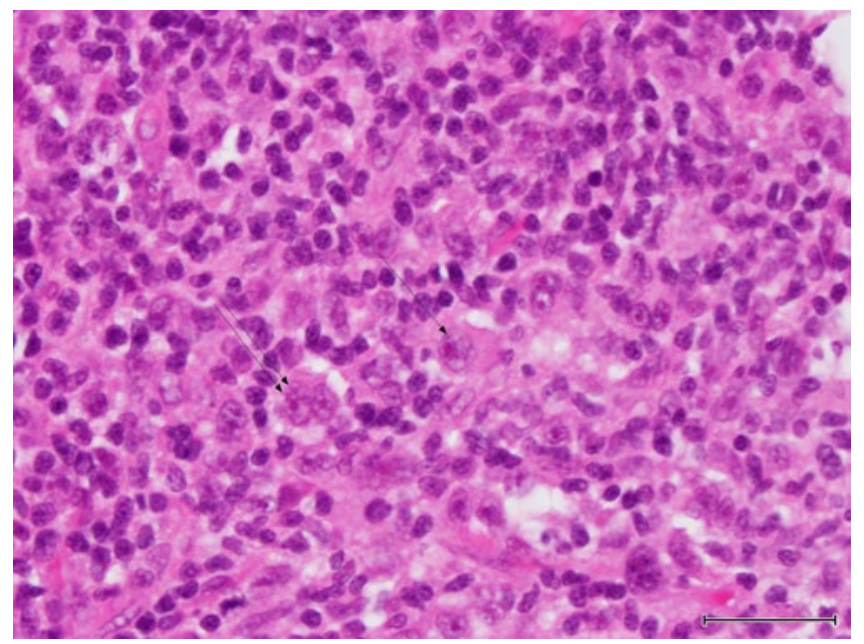

Abb. $5<$ Detailaufnahme mit einer Hodgkin-Zelle (Pfeil) und einer mehrkernigen Reed-SternbergZelle (Doppelpfeil), beide teilweise rosettenartig von Lymphozyten umgeben (HE-Färbung, Vergrößerung 400-fach, Balken entspricht $50 \mu \mathrm{m}$ )

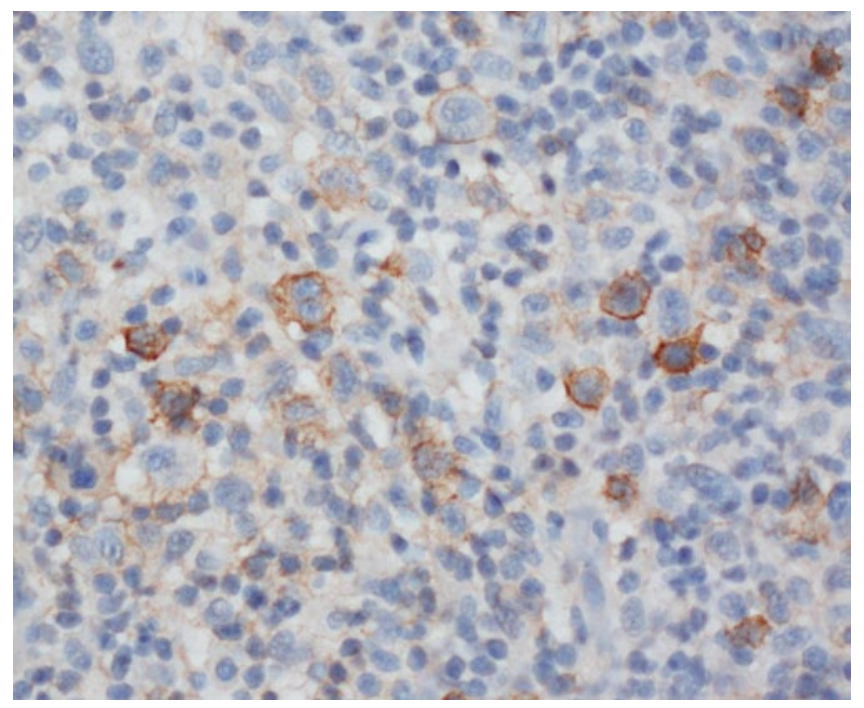

Abb. $6<$ Nachweis der CD30-Expression in Hodgkin- und ReedSternberg-Zellen (Peroxidasereaktion, Vergrößerung 400-fach)

Darmerkrankungen (CED), welche häufig unter immunsuppressiver Medikation stehen, eigentlich dieser erst kürzlich beschriebenen Entität zuzuordnen wären $[3,7]$. Bei der hier beschriebenen Patientin war keine Immunsuppression im en- geren Sinne bekannt und die Patientin litt auch nicht unter einer CED. Allerdings bestand bei der Patientin eine Reihe chronischer und therapiebedürftiger Grundleiden, sodass die Immunlage möglicherweise dennoch insgesamt eingeschränkt war und es denkbar ist, dass eine lokale EBV-Reaktivierung mit zur Pathogenese des Lymphoms beigetragen haben könnte. Die differenzialdiagnostische Abgrenzung gegenüber einem „EBV-assoziierten diffus großzelligen B-Zell-Lymphom des älteren Patienten“, für welches Hodgkinähnliche Merkmale beschrieben werden [8], gelingt im vorliegenden Fall über den stark herabreguliertem B-Zell-Phänotyp, fehlenden Nachweis von Nekrosen sowie die durchgängige Pauzizellularität des Infiltrats [1].

Aufgrund der abschließenden Staginguntersuchungen wurde der Befund als Stadium IE festgelegt. Die Therapie dieser Lymphomgruppe wird auch bei den kolorektalen Lymphomen kontrovers diskutiert. Belegt ist der positive Effekt operativer Eingriffe (chirurgische Resektion mit negativen Resektionsrändern). Während für das Stadium II der Nutzen einer adjuvanten Chemotherapie demonstriert wurde (117,4 vs. 47,9 Monate [5]), konnte dies für das auch hier vorliegende lokale Stadium nicht belegt werden $[5,6]$. Zusammen mit der spezifischen Krankheitsgeschichte der hier beschriebenen Patientin (zahlreiche chronische Vorerkrankungen und fortgeschrittenes Alter) wurde daher zunächst auf eine systemische Therapie verzichtet, von der Studienzentrale jedoch abschließend eine Chemotherapie empfohlen. Aufgrund der extrem niedrigen Quoten primärer kolorektaler Hodgkin-Lymphome sind statistisch gesicherte Daten anhand größerer Kohorten nicht zu erwarten, sodass die Therapieentscheidung weiterhin primär fallbezogen bleiben dürfte und in Absprache mit einer Studienzentrale erfolgen sollte.

\section{Fazit für die Praxis}

- Maligne Lymphome machen weniger als $1 \%$ aller Kolonneoplasien aus; hierunter sind Hodgkin-Lymphome umso mehr als echte Raritäten einzustufen.

- Der klinische Befund ist uncharakteristisch; bei exulzerierten Lymphomen können oberflächliche Biopsate u. U. keine definitive Diagnose erbringen.

- Primär intestinale EBV-assoziierte Hodgkin-Lymphome sind insbesondere differenzialdiagnostisch von der 


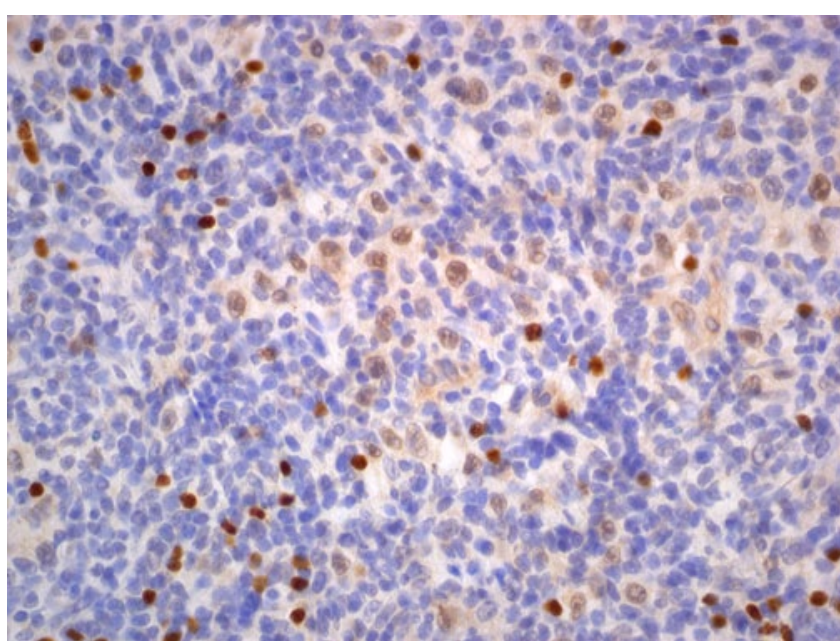

Abb. $7<$ Nachweis der PAX-5-Detektion mit typischer schwacher Kernanfärbung der Hodgkin- Blasten und der kräftigen Kernanfärbung der residuellen B-Lymphozyten (Peroxidasereaktion, Vergrößerung 400-fach)

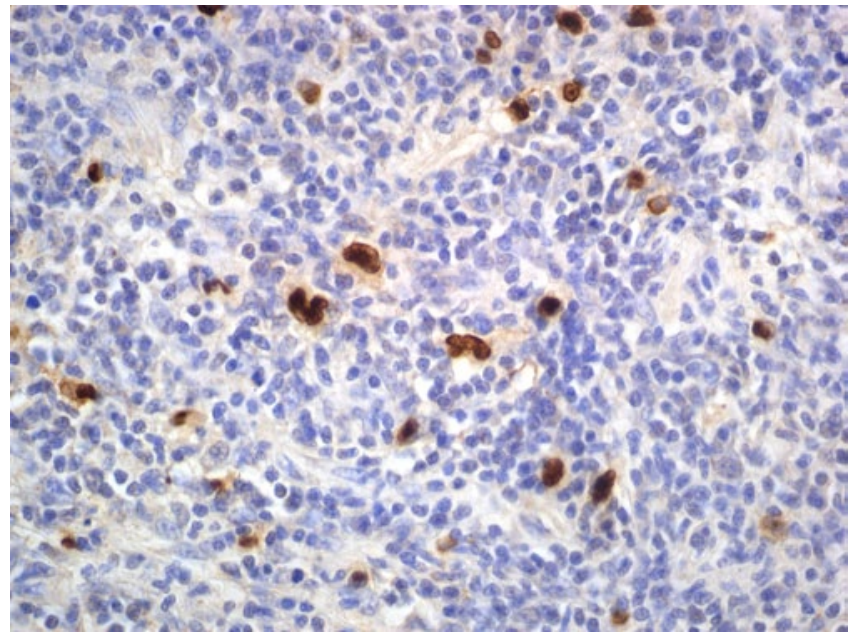

Abb. $8<$ EBV-Nachweis mittels In-situHybridisierung (Peroxidasereaktion, Vergrößerung 400-fach).
3. Dojcinov S, Venkataraman G, Raffeld M et al (2010) EBV positive muocutaneous ulcers - a study of 26 cases associated with various sources of immunosuppression. Am J Surg Pathol 34:405-417

4. Doolabh N, Anthony T, Simmang Cet al (2000) Primary colonic lymphoma. J Surg Oncol 74:257-262

5. Fan CW, Changchien CR, Wang JY et al (2000) Primary colorectal lymphoma. Dis Colon Rectum 43:1277-1282

6. Gonzalez $\mathrm{OH}$, Heslin MJ, Dávila-Cervantes A et al (2008) Primary colonic lymphoma. Am Surg 74:214-216

7. Kumar S, Fend F, Quintanilla-Martinez L et al (2000) Epstein-Barr virus-positive primary gastrointestinal Hodgkin's disease: association with inflammatory bowel disease and immunosuppression. Am J Surg Pathol 24:66-73

8. Nakamura S, Jaffe ES, Swerdlow SH (2008) EBV positive diffuse large $B$ cell lymphoma of the elderly. In: Swerdlow SH, Campo E, Harris NL et al (eds) WHO classification of tumours of haematopoietic and lymphoid tissues. IARC Press, Lyon, pp 243244

9. Piccaluga PP, Agostinelli C, Gazzola A et al (2011) Pathobiology of Hodgkin lymphoma. Adv Hematol. doi:10.1155/2011/920898

10. Stanojevic GZ, Nestorovic MD, Brankovic BR et al (2011) Primary colorectal lymphoma: an overview. World J Gastrointest Oncol 3:14-18

11. Vadmal MS, LaValle GP, De Young BR et al (2000) Primary localized extranodal Hodgkin disease of the transverse colon. Arch Pathol Lab Med124:1824-1827
„EBV-positiven mukokutanen Ulzeration" abzugrenzen; dies kann aufgrund sehr ähnlicher histopathologischer Merkmale der Entitäten nur über eine klinisch-pathologische Korrelation erfolgen.

- Für die Diagnose des klassischen HL allgemein sind neben der typischen Histomorphologie der immunhistochemische Nachweis von CD30 und PAX-5 bedeutsam, die Expression für CD15 kann hingegen (wie im vorliegenden Fall) fehlen.

- Während der prognostisch positive Effekt der chirurgischen Resektion belegt ist, wird der Einsatz adjuvanter Chemotherapie bei rein lokalem Lymphombefall kontrovers diskutiert; da statistisch gesicherte Daten aufgrund der Seltenheit primärer kolorektale Hodgkin-Lymphome fehlen, sollte die Therapieentscheidung durch einen erfahrenen Onkologen bzw. in Ab- sprache mit einer Studienzentrale fallbezogen erfolgen.

\section{Korrespondenzadresse}

PD Dr. T. Hansen

Institut für Pathologie, Klinikum Lippe $\mathrm{GmbH}$, Detmold

Röntgenstr. 18, 32756 Detmold

Torsten.Hansen@klinikum-lippe.de

Interessenkonflikt. Der korrespondierende Autor gibt für sich und seine Koautoren an, dass kein Interessenkonflikt besteht.

\section{Literatur}

1. Asano N, YamamotoK, Tama Jl et al (2009) Agerelated EBV-associated B-cell lymphoproliferative disorders: comparison with EBV-positive classic Hodgkin lymphoma in elderly patients. Blood 113:2629-2636

2. Bautista-Quach MA, Ake CD, Chen M, Wang J (2012) Gastrointestinal lymphomas: morphology, immunophenotype and molecular features. J Gastrointest Oncol 3:209-225

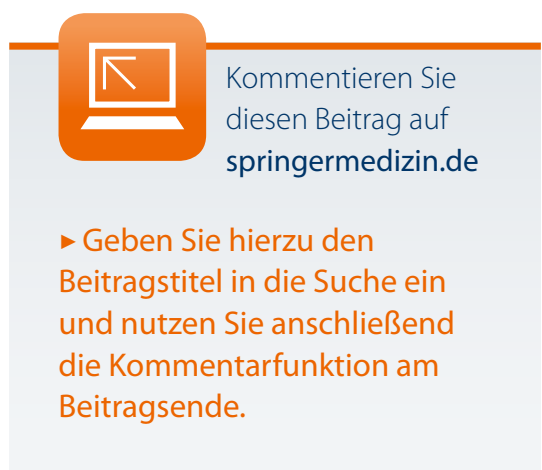

